\title{
Drosophila ML-DmD17-c3 cells respond robustly to Dpp and exhibit complex transcriptional feedback on BMP signaling components
}

\author{
Scott J. Neal ${ }^{1 *+} \mathbb{B}$, Darin Dolezal ${ }^{1,2,4+}$, Nisveta Jusić ${ }^{1}$ and Francesca Pignoni ${ }^{1,2,3^{*}}$
}

\begin{abstract}
Background: BMP signaling is involved in myriad metazoan developmental processes, and study of this pathway in Drosophila has contributed greatly to our understanding of its molecular and genetic mechanisms. These studies have benefited not only from Drosophila's advanced genetic tools, but from complimentary in vitro culture systems. However, the commonly-used S2 cell line is not intrinsically sensitive to the major BMP ligand Dpp and must therefore be augmented with exogenous pathway components for most experiments.

Results: Herein we identify and characterize the responses of Drosophila ML-DmD17-c3 cells, which are sensitive to Dpp stimulation and exhibit characteristic regulation of BMP target genes including Dad and brk. Dpp signaling in ML-DmD17-c3 cells is primarily mediated by the receptors Put and Tkv, with additional contributions from Wit and Sax. Furthermore, we report complex regulatory feedback on core pathway genes in this system.

Conclusions: Native ML-DmD17-c3 cells exhibit robust transcriptional responses to BMP pathway induction. We propose that ML-DmD17-c3 cells are well-suited for future BMP pathway analyses.

Keywords: Bone morphogenetic protein (BMP), Decapentaplegic (Dpp), Schneider (S2) cells, ML-DmD17-c3 cells, Phospho-mad (pMad), Daughters against Dpp (Dad), Punt (Put), Thickveins (Tkv), Wishful thinking (Wit), Saxophone (Sax)
\end{abstract}

\section{Background}

The Bone Morphogenetic Protein (BMP) signaling pathway plays key roles in metazoan development and stem cell maintenance, at wound healing sites, and in myriad other contexts [1-3]. In Drosophila the BMP signaling cascade is less complex [4], whereas in mammals it features many specialized or redundant elements. Some of the pioneering work in discovering fundamental molecular and cellular mechanisms of BMP signaling has been conducted in the fly [5-7], and this continues to be an active area of research as new BMP signaling modulators are identified [8]. Thus, the simpler Drosophila system represents an ideal paradigm in which to elucidate

\footnotetext{
*Correspondence: neals@upstate.edu; pignonif@upstate.edu

${ }^{\dagger}$ Scott J. Neal and Darin Dolezal contributed equally to this work.

${ }^{1}$ Center for Vision Research and Department of Ophthalmology, Upstate

Medical University, NRB-4610, 505 Irving Ave, Syracuse 13210, NY, USA

Full list of author information is available at the end of the article
}

mechanistic contributions of core BMP pathway components and modulators.

In Drosophila there are three BMP-like ligands encoded by the genes decapentaplegic $(d p p)[6,9,10]$, glass bottom boat (gbb) [11, 12], and screw (scw) [13], of which Dpp is the best characterized and has been shown to play diverse developmental roles. Signaling initiates upon ligand binding to one of the constitutive Type II BMP receptors (Punt - Put, or Wishful Thinking - Wit) which in turn associates with, and transactivates, one of the Type I BMP receptors (Thickveins - Tkv, or Saxophone - Sax) [14, 15]. The activated receptor complex recruits and phosphorylates an intracellular signal transduction component, the receptor-regulated R-SMAD transcription factor Mad (Mothers against DPP) [16]. Phosphorylated Mad (pMad) associates with the co-SMAD Medea (Med) and together they translocate

(c) The Author(s). 2019 Open Access This article is distributed under the terms of the Creative Commons Attribution 4.0 International License (http://creativecommons.org/licenses/by/4.0/), which permits unrestricted use, distribution, and reproduction in any medium, provided you give appropriate credit to the original author(s) and the source, provide a link to the Creative Commons license, and indicate if changes were made. The Creative Commons Public Domain Dedication waiver (http://creativecommons.org/publicdomain/zero/1.0/) applies to the data made available in this article, unless otherwise stated. 
into the nucleus to regulate gene expression [17-20]. Among the transcriptional targets of Dpp signaling are genes encoding the inhibitory I-SMAD Dad (Daughters Against Dpp) [17, 21], and downstream mediators of the response to Dpp such as Bam (Bag of Marbles) [22], and Brk (Brinker) [23-25]. The simplicity of the cascade and the powers of genetic manipulation in Drosophila render the fruit fly a premier system for the study of fundamental aspects of BMP signaling in vivo.

The strength of the in vivo analyses in this animal model has been increased by in vitro experiments in cell culture that have investigated the pathway at a biochemical level using some of the earliest Drosophila cell lines, the Schneider (S2) line [9, 11, 16, 26-32], and Kc167 cells [33]. In particular, S2 cells have been invaluable in elucidating a variety of basic properties of BMP signal transduction, although they are not inherently responsive to Dpp. S2 cells are routinely augmented via supplementation of pathway components (e.g. constitutively-activated Tkv receptor or exogenous Mad transducer) to evaluate signaling activity [16, 28-32]. Furthermore, diverse S2 isolates with drastically different transcriptomes are in use throughout the community [34], making it difficult to reconcile published results pertaining to pathway activity and modulation.

In this study, we investigated several molecularly characterized Drosophila cell lines [34] to select one more suited to BMP pathway analysis. We found the ML-DmD17-c3 cell line [35] to be inherently responsive to the Dpp ligand across a wide range of concentrations. We demonstrate the respective contributions of the four BMP receptors to signaling, and examine the intricate transcriptional feedback that results from pathway activation in these cells. Absent any augmentation, ML-DmD17-c3 cells recapitulate key aspects of BMP signaling in vivo and therefore represent a valuable alternative tool for mechanistic studies of this essential signaling pathway.

\section{Results}

Identification of ML-DmD17-c3 cells and characterization of their responsiveness to Dpp stimulation

Leveraging the transcriptome datasets produced by the modENCODE project $[34,36]$, we selected three candidate cells lines (ML-DmD4-c1; ML-DmD8; ML-DmD17-c3; [35]) with the highest transcript levels of key components of the Dpp signal transduction cascade (particularly $t k v, M a d$, and Med) (Fig. 1a, Additional file 1: Table S1). For comparison, we examined the established S2-DRSC ('S2' hereafter) and related S1 cell lines [26, 27, 34], as well as the central nervous system-derived ML-DmBG2-c2 cells [37]. Each cell culture was stimulated with $5 \mathrm{nM}$ exogenous Dpp and transcript levels of the positively-regulated gene target
Dad were measured by reverse transcription-quantitative (rt-q)PCR (Fig. 1b). ML-DmD4-c1 and ML-DmD17-c3 cells exhibited approximately 4-fold greater induction of Dad transcript than either S1 or S2 cells. Induction of Dad expression in ML-DmD8 reached an intermediate level, higher than in S2 but lower that in ML-DmD17-c3 cells. Lastly, expression of Dad was not affected by Dpp in ML-DmBG2-c2 cells; a result consistent with a failure to respond due to low expression of critical cascade components (Additional file 1: Table S1).

In particular, ML-DmD17-c3 cells ('D17' hereafter) displayed maximal relative Dad induction as well as the highest combination of $t k v, \mathrm{Mad}$ and Med expression [34]. To independently assess the robustness of this cell line's response to Dpp, we measured pathway activation using a luciferase reporter driven by the minimal activating enhancer for the Dad locus [38]. We again observed increased pathway activation in D17 cells compared with S2 cells; a $\sim 40$-fold relative induction of luciferase activity in D17 cells stimulated with $5 \mathrm{nM}$ Dpp as compared with a 10-fold induction in S2 cells (Fig. 1c). Thus, D17 cells exhibited a similar magnitude of enhancement (4-fold) over S2 cells in the Dad response using the synthetic reporter construct as for endogenous transcript levels. Based on these observations we elected to further characterize the D17 cell line.

In addition to activating transcription, Dpp signaling acts via "silencer elements" to repress gene expression [39]. This has been extensively documented at the bam and brk loci [5, 39-42]. Hence, we sought to confirm this expected response to pathway induction in our experimental system. We found the expression of both genes to be consistently and robustly repressed by Dpp treatment in D17 cells (Fig. 1d).

Next, we explored the dose-response characteristics of D17 cells, as concentrations from $1 \mathrm{pM}$ to $4 \mathrm{nM}$ exogenous Dpp have been reported for experiments in S2 cells $[29,31,32,43,44]$. We tested Dpp concentrations from $0.1 \mathrm{nM}$ to $10 \mathrm{nM}$ and measured the Dad transcriptional response of D17 cells by rt-qPCR (Fig. 1e). We observed statistically significant Dad induction using as little as $0.25 \mathrm{nM}$ Dpp and an essentially maximal response by $7 \mathrm{nM}$.

Lastly, Dpp pathway induction in S2 cells has been previously assessed by immunodetection of nuclear phosphorylated Mad (pMad) in cells [16, 31, 32, 45], and by Western blot detection of pMad from lysates of cells stimulated with as little as $10 \mathrm{pM}$ Dpp [31]. Therefore, we sought to confirm that we could detect nuclear pMad accumulation in the D17 cell line by immunocytochemistry. Only sporadic D17 cells exhibited nuclear accumulation of pMad under the unstimulated condition (no Dpp) while those stimulated with $5 \mathrm{nM}$ Dpp exhibited clear nuclear pMad accumulation (Fig. 1f-g). 


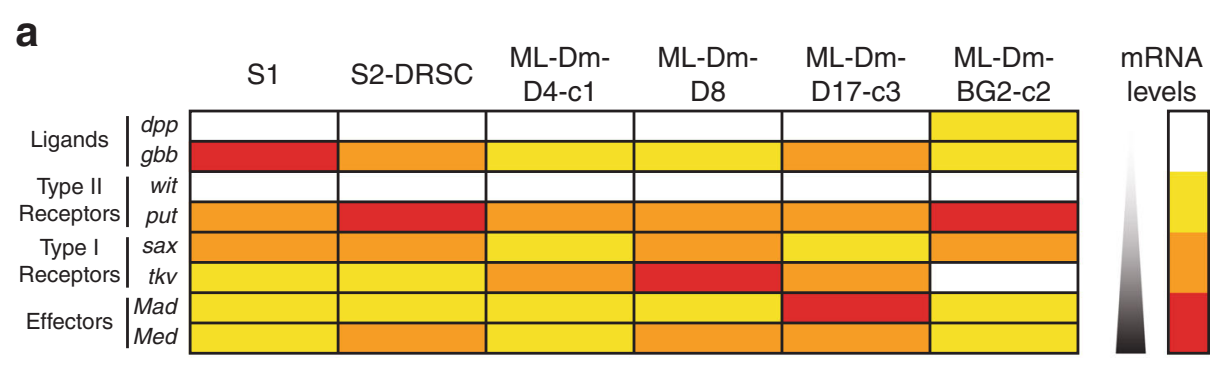

b

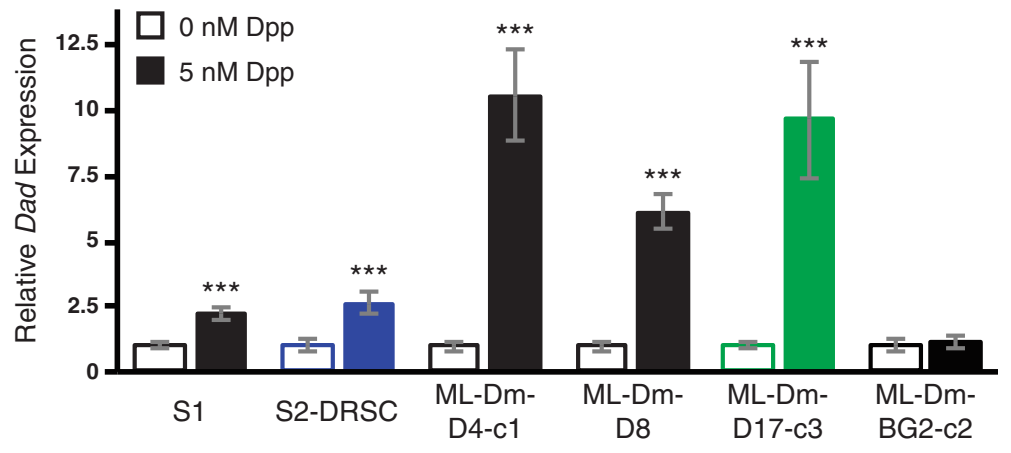

C

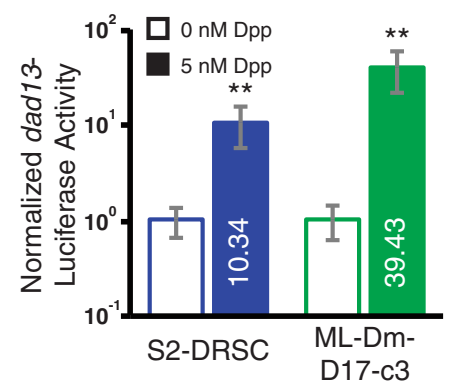

d

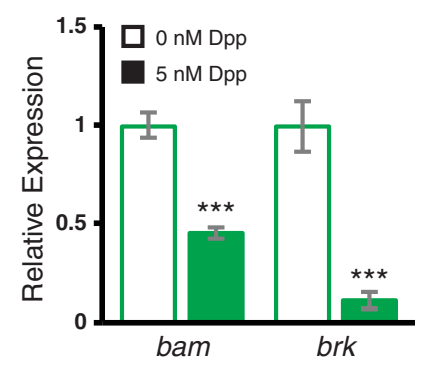

e

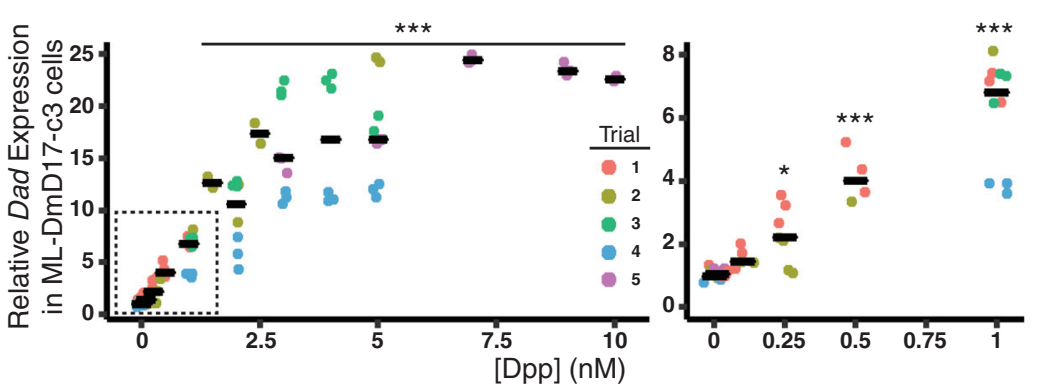

f

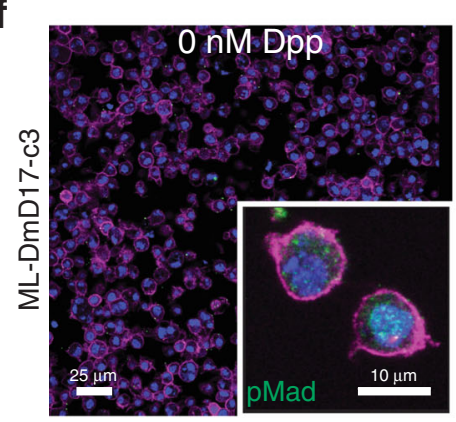

g

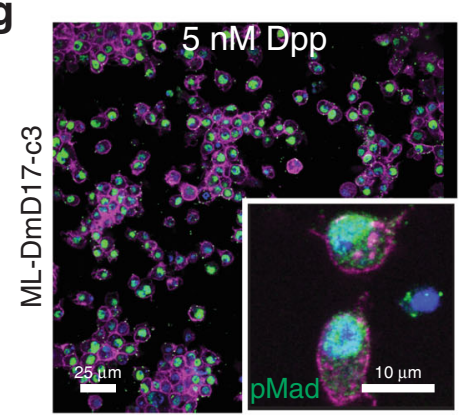

Fig. 1 (See legend on next page.) 
(See figure on previous page.)

Fig. 1 Identification of ML-DmD17-c3 (D17) cells, and characterization of their responsiveness to Dpp stimulation. (a) Graphical representation of gene expression values derived from modENCODE data [34] for each of six cell lines used in this study. The functional category and respective genes are listed to the left. Those with low (500-1000, yellow), medium (1000-2000, orange) and high (> 2000, red) expression are shaded proportionally to their expression values within each category. Expression values below 500 units are considered unreliable (white). It is only appropriate to compare expression values across cell lines within a gene, and not between genes (https://dgrc.bio.indiana.edu/cells/ TilingDescription). (b) Quantification of relative Dad expression, normalized to Act5C expression, for each of the six cell lines used in this study, in the absence (empty bars) or presence (filled bars) of $5 \mathrm{nM}$ recombinant Dpp. Baseline expression within each cell line was scaled to 1. Values given represent the mean and standard deviation of two independent assays, each with $2-3$ technical replicates. $P$ values were calculated for the pairwise comparison of means using Student's t-test; *** $P<0.001$. (c) Quantification of relative dad13-Iuciferase activity, normalized to CMVRenilla activity, for S2 and D17 cells, in the absence (empty bars) or presence (filled bars) of $5 \mathrm{nM}$ recombinant Dpp. Baseline expression was scaled to 1 for each cell line, and the fold-induction of dad13-luciferase activity is given within the filled bars; note the logarithmic axis. Values given represent the mean and standard deviation of two independent assays, each with 2-3 technical replicates. $P$ values were calculated for the pairwise comparison of means using Student's t-test; ${ }^{* *} P<0.01$. (d) Quantification of relative bam and brk expression, normalized to Act5C expression, in D17 cells, in the absence (empty bars) or presence (filled bars) of $5 \mathrm{nM}$ recombinant Dpp. Baseline expression was scaled to 1 for each gene. Values given represent the mean and standard deviation of two independent assays, each with 2-3 technical replicates. $P$ values were calculated for the pairwise comparison of means using Student's t-test; ${ }^{* *} P<0.001$. (e) Quantification of relative Dad expression, normalized to Act5C expression, in D17 cells treated with the indicated concentrations of recombinant Dpp. Each assay is represented by a filled circle and independent assays are grouped by color, as indicated; median responses are indicated by black horizontal bars. The region contained within the dashed box is expanded to the right of the primary graph. Note that we observed larger variance between trials than within trials; we cannot explicitly account for these differences at this time. Data were analyzed using a general linear model using SPSS (IBM) and "Trial" was treated as a random factor. A posthoc Bonferroni test was used to calculate pairwise $P$ values; ${ }^{*} P<0.05$, ${ }^{* *} P<0.01$, ${ }^{* *} P<0.001$. (f, g) Representative images of immunocytochemical detection of pMad (green), cytoskeleton (magenta) and nuclei (blue) of untreated D17 cells (f) and of those treated with $5 \mathrm{nM}$ recombinant Dpp (g) at low magnification and high magnification (insets)

In conclusion, the ensemble of experiments we have conducted demonstrate that native D17 cells exhibit all the hallmarks of robust BMP signaling.

\section{All four BMP receptors contribute to Dpp signaling in D17 cells}

To further examine the molecular mechanism of Dpp signal transduction in D17 cells, we assessed the contributions of the various Type I and II receptors. In S2 cells, Dpp-induced responses reflect the function of Tkv and Put, but not Sax [31]. Using the RNAi soaking method for Drosophila cultured cells [46, 47], we individually knocked-down (KD) each of the four receptor genes, sax, $t k v$, put and wit (Additional file 2: Figure S1a). Under Dpp-induced conditions, put or $t k v$ KD almost-completely abrogated Dad induction as well as blocked brk repression (Fig. 2a-b). D17 cells were particularly sensitive to KD of either put or $t k v$; reducing transcript levels by as little as $20 \%$ resulted in a detectable decrease in maximal Dad induction (Additional file 2: Figure S1b-c). Furthermore, we observed a graded relationship between residual steady-state put or $t k v$ transcript levels and Dpp-induced Dad transcriptional output (Additional file 2: Figure S1b-c). Maximal RNAi-mediated KD of put or $t k v$ reduced the basal expression of $\mathrm{Dad}$ in unstimulated cells by 35 to $50 \%$, respectively (Fig. 2c), but did not result in significant de-repression of brk (Fig. 2d). This observation suggests there is tonic BMP signaling in unstimulated D17 cells that contributes to the steady-state expression level of Dad but not brk.

Interestingly, KD of sax or wit yielded small yet significant reductions in Dad activation (by $25 \%$ and $30 \%$, respectively), but had no effect on brk repression (Fig. $2 a-b)$, thus revealing a differential response by these two gene targets, consistent with our observations in unstimulated cells. A priori, we did not expect to observe an effect of wit KD on pathway activity because modENCODE data showed wit expression to be in the low/unreliable range in D17 cells. However, as shown (Additional file 2: Figure S1d) and elaborated on below, Dpp-induction leads to enhanced expression of wit mRNA. Hence, the observed consequences of wit KD likely underscore a contribution of Wit to pathway activity.

In short, the transduction of the Dpp signal across the membrane of D17 cells is mediated primarily by the receptors Tkv and Put, with lesser contributions from Sax and Wit receptors.

\section{Transcription of pathway components is auto-regulated in Dpp-treated D17 cells}

BMP signaling is subject to multiple levels of regulation [5, 48], which allow for it to function in diverse biological contexts. Autoregulation is essential in order to produce responses that are sequential or graded in space and time and, as mentioned in the introduction, two of the best characterized direct BMP pathway gene targets, Dad and brk, encode factors with autoregulatory activity $[21,25]$. Dad functions as an I-SMAD, targeting Tkv for degradation [17, 21, 49]; Brk, a transcriptional repressor, competes with Mad for binding to certain gene regulatory sites $[30,38,50]$. Interestingly, the regulation of these factors in response to pathway induction is discordant. The strong upregulation of the signaling 


\section{a}

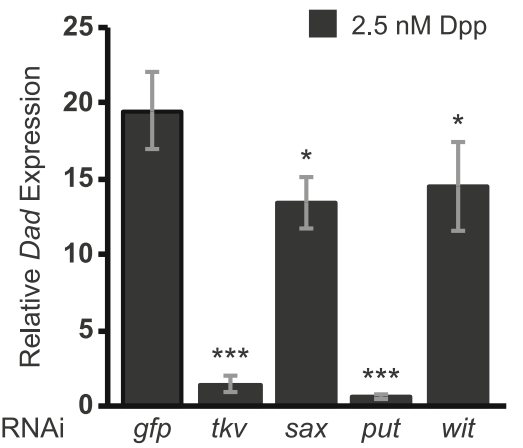

C

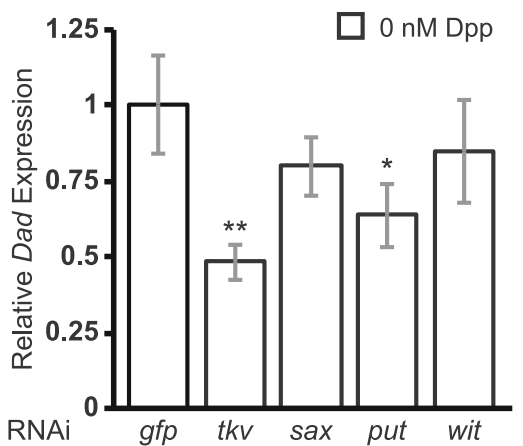

b

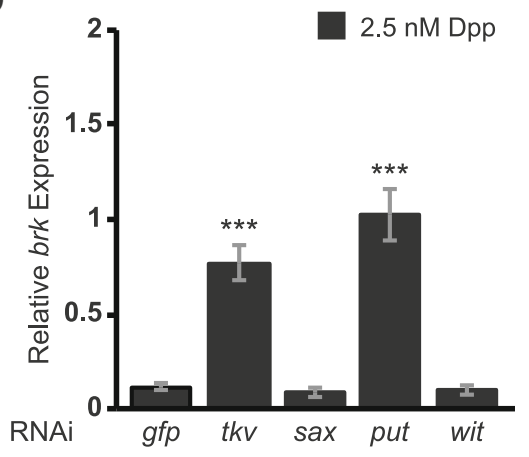

d

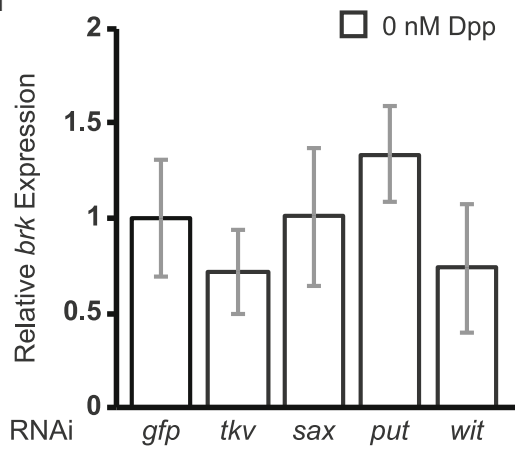

Fig. 2 Put and Tkv are the primary transducers of Dpp signaling in ML-DmD17-c3 cells. Relative Dad (a, c) and brk (b, d) expression, normalized to Act5C expression, was measured in D17 cells treated with control ( $g f p)$ or receptor ( $t k v$, sax, put, wit) RNAi, as indicated, in the presence (a, $\mathbf{b}$; filled bars) and absence (c, $\mathbf{d}$; open bars) of $2.5 \mathrm{nM}$ recombinant Dpp. Values given represent the mean and standard deviation of two independent assays, each with 2-3 technical replicates. Data were analyzed using a general linear model in SPSS (IBM) and "Trial" was treated as a random factor. A posthoc Bonferroni test was used to calculate pairwise $P$ values; ${ }^{*} P<0.05$, * $P<0.01$, *** $P<0.001$

attenuator encoded by Dad contrasts with the reduced expression of the transcriptional repressor encoded by brk. Such "incoherent feedback" has been observed in several signaling pathways [43], and may be important for the capacity of a simple signaling cascade to generate diverse outputs $[51,52]$.

Thus, we explored the modulation of core pathway component expression as a potential additional level of feedback regulation. Specifically, we analyzed the mRNA level of the ligands $d p p$ and $g b b$ (Fig. 3a-b, respectively), the receptors $t k v$, sax, put and wit (Fig. 3c-f, respectively), and the intracellular transducers Mad and Med (Fig. 3g-h, respectively), in D17 cells treated with different concentrations of exogenous Dpp $(1,3$, or $5 \mathrm{nM})$. We observed a significant reduction of $d p p$ expression in all treatment conditions $\left(P_{\text {Dpp }}=0.037, P_{\text {Trial }}=0.015\right.$, $\left.P_{\text {Dpp"Trial }}=0.369\right)($ Fig. 3a), while the mRNA level of $g b b$ was unaffected (Fig. $3 \mathrm{~b}$ ). With respect to the receptors, we observed a small but significant Dpp-dependent increase in the expression of $t k v$ in cells stimulated with 5 nM Dpp $\left(P_{\text {Dpp }}=0.036, P_{\text {Trial }}<0.001, P_{\text {Dpp }}\right.$ Trial $=0.426$; Fig. 3c). Small effects on sax and put transcripts were observed in only one of the three trials (Fig. 3d, e), whereas wit mRNA levels were consistently and dramatically upregulated in a Dpp dose-dependent manner $\left(P_{\text {Dpp }}=0.001, P_{\text {Trial }}=0.179, P_{\text {Dpp } * \text { Trial }}=0.002\right.$; Fig. 3f). Downstream of the receptors, the expression of $\mathrm{Mad}$ was downregulated by each concentration of Dpp tested $\left(P_{\text {Dpp }}<0.001, P_{\text {Trial }}<0.001, P_{\text {Dpp }}\right.$ "Trial $=0.965$; Fig. $3 g$ ), whereas that of $\mathrm{Med}$ showed only slight repression with $3 \mathrm{nM}$ Dpp $\left(P_{\mathrm{Dpp}}=0.144, P_{\text {Trial }}=0.022\right.$, $P_{\text {Dpp*Trial }}=0.132$; Fig. $3 \mathrm{~h}$ ).

In summary, D17 cells modulate the expression of genes encoding BMP ligand $(d p p)$, receptors $(t k v$, wit) and transducer $(\mathrm{Mad})$ in response to BMP pathway activation by exogenous Dpp. The lowering of $d p p$ and Mad mRNAs is anticipated to decrease pathway activity (negative feedback), whereas the dramatic increase in wit mRNA could reflect a compensatory loop that serves to sustain pathway activity in some contexts. Regardless, these effects suggest that complex mechanisms, beyond the direct regulation of factors such as Dad and brk, are at work to tailor BMP pathway activity, even within a simple cell culture system.

\section{Discussion}

We have characterized the Dpp response of D17 cells and have shown that they exhibit robust and diverse 

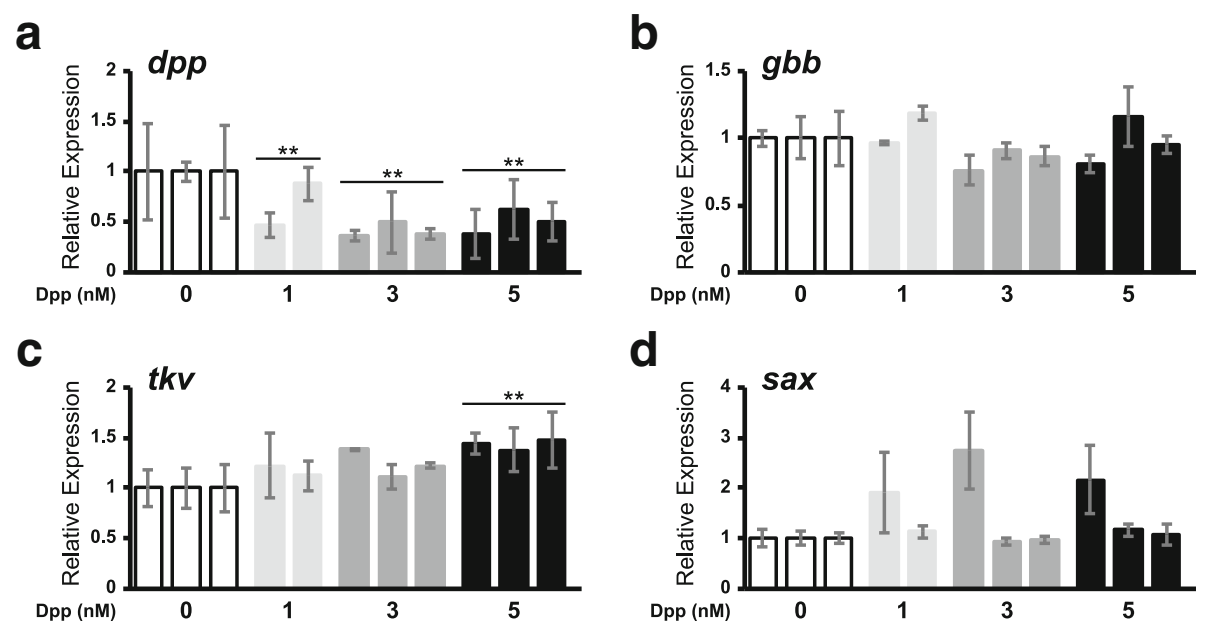

d

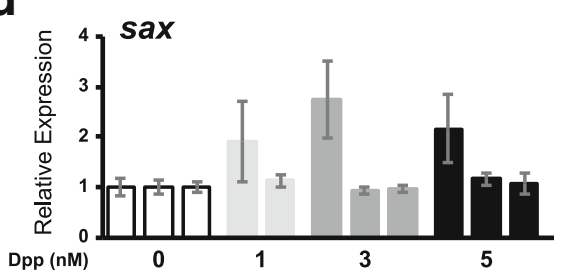

e

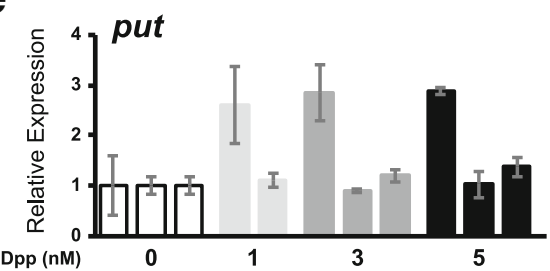

f

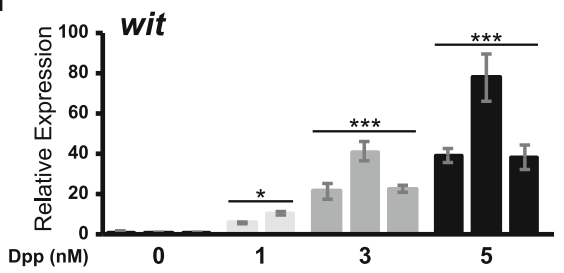

9

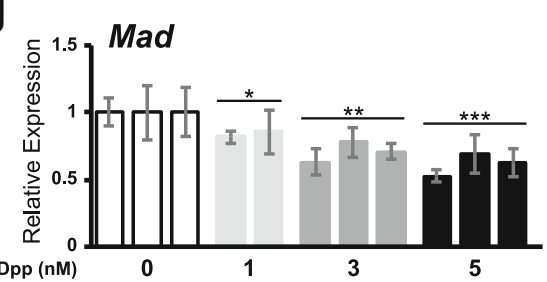

h

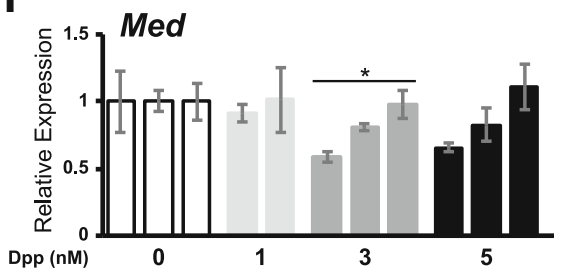

Fig. $\mathbf{3}$ Transcription of pathway components is auto-regulated in Dpp-treated ML-DmD17-c3 cells. Relative expression of the indicated genes (a-h), normalized to Act5C expression, was measured in untreated cells (white bars) and in those treated with $1 \mathrm{nM}, 3 \mathrm{nM}$ or $5 \mathrm{nM}$ recombinant Dpp (light gray, medium gray, and black, respectively). The Y-axes are scaled to the maximum expression for each gene. Each bar represents the mean and standard deviation of a single assay, consisting of 2-3 technical replicates. Baseline gene expression in untreated cells was normalized to 1 within each trial and the order of trials is maintained across treatments; "Trial 3" for $1 \mathrm{nM}$ Dpp was not completed. Data were analyzed using a general linear model in SPSS (IBM) and "Trial" was treated as a random factor. A posthoc Bonferroni test was used to calculate pairwise $P$ values; ${ }^{*} P<0.05$, ** $P<0.01,{ }^{* * *} P<0.001$

transcriptional responses to exogenous Dpp stimulation. These effects are primarily mediated by Put and Tkv, with additional contributions from Sax and Wit, and result in feedback-regulation of core pathway genes $d p p$, $t k v$, wit and Mad. In addition, these cells are amenable to the passive RNAi soaking method [46, 47, 53], facilitating RNAi-based approaches. Thus, D17 cells offer a paradigm that is uncomplicated by potential artifacts and limitations associated with manipulating pathway component levels by transfection. We believe that D17 cells are well-suited to analyses of constitutive and nuanced contributions of known and novel BMP pathway components to signaling output.

D17 cells were isolated from haltere imaginal discs [35], and exhibit a transcriptome consistent with the haltere hinge region [34]; the hinge/notum boundary is a region in the closely-related wing imaginal disc where Dpp signaling is essential $[54,55]$. More recently it has been proposed that D17 cells exhibit haemocyte-like properties, including motility and phagocytosis [56]. However, D17 cells exhibit low $d p p$ expression, unlike haemocytes [57, 58], and also form cell-cell junctions [56]. Origin aside, the results herein demonstrate that D17 cells are a versatile system for the study of BMP signaling.

In vivo, Tkv and Put play the major role in Dpp-initiated signaling, but different contexts and ligands also provide evidence of BMP signaling through the other receptors, Sax and Wit [59-63]. Tkv and Sax show partial functional overlap as well as distinct phenotypes [59]; in certain contexts, double-mutant combinations of $t k v$ and sax display more severe phenotypes that more closely 
resemble $d p p$ loss of function [59]. In the embryo, it was suggested that Sax may respond to only high levels of Dpp whereas Tkv functions at lower Dpp ligand levels [64]. Alternatively, within the context of a gradient of BMP signaling in the wing, it has been suggested that constitutive Gbb/Sax signaling serves to enhance Dpp/ Tkv signaling where the latter is at low levels [61]. The contributions of Sax and Wit to BMP signal transduction at different ligand concentrations could be explored using the D17 cell model system. Moreover, Sax has been shown to respond to the Gbb and Scw ligands in some biological processes, including wing development and embryonic ectoderm development, respectively [61, $65,66]$, and D17 cells could also be used to assess the interplay among these ligands and receptors. Overall, these results suggest that the combined action of Tkvand Sax-containing BMP receptors is necessary to fully decode the Dpp signal in vivo. In addition, Sax- and Wit- containing receptor complexes play principal roles in responding to the $\mathrm{Scw}$ and $\mathrm{Gbb}$ ligands, respectively $[62,65,67,68]$.

In response to Dpp stimulation, we observed decreased expression of $d p p$, although $d p p$ expression was low to begin with. Dpp-dependent $d p p$ regulation has been observed in multiple instances in vivo $[61,69,70]$, and in vitro [43], yet the context ultimately determines whether these effects are positive or negative. In vivo, elevated expression of $\mathrm{Tkv}$ and constitutively-active $\mathrm{Tkv}$ were shown to downregulate $d p p$ expression $[61,69]$, a paradigm likely mimicked by long-term $(18-24 \mathrm{~h})$ exposure of cells to exogenous Dpp (this study). On the contrary, short-term (30-60 min) exposure of S2 cells to Dpp resulted in increased $d p p$ expression [43]; in vivo, $d p p$ induction was also observed when Dpp was ectopically expressed in the anterior of developing eye imaginal disc [70]. Hypotheses regarding the temporal and mechanistic control of $d p p$ expression, such as evaluating direct (pMad-dependent) versus indirect (secondary) BMP signaling effects, could be further explored in D17 cells.

In regards to the regulation of Mad and wit, our results raise the possibility of additional levels of pathway autoregulation. For instance, a recent report has shown that wit expression is indirectly regulated through the relief of Brk repression [71]. Investigating the transcriptional (or post-transcriptional) mechanisms for the observed changes in Mad and wit mRNA levels, and identifying relevant in vivo contexts for this regulation, represent avenues for future research. Altogether, these effects are predicted to both enhance (increased wit) and repress (decreased $d p p$ and Mad) BMP signaling, providing additional evidence of incoherent feedback, as discussed above for Dad and brk. D17 cells may be particularly useful in dissecting how such discordant inputs are integrated to set a specific level of BMP signaling.
Several outstanding questions regarding the integrated response of cells to stimulation by BMPs remain unanswered. For instance, BMP receptors are thought to exist as heterotetramers [1], and little is known of how the composition of such complexes affects signal transduction, specifically as it pertains to that of distinct ligand heterodimers [11, 61, 68]. In addition, we have demonstrated that depleting specific BMP receptors results in differential effects on known direct transcriptional targets ( $D a d, b r k)$. Given the evidence of activity for each of the four receptors in D17 cells, it may be possible to investigate the intricacies of BMP receptor interplay using this system. Such an analysis could benefit from examining a more comprehensive set of target genes and by using a more parallel approach, for example Nanostring technology [43].

One potential advantage of D17 cells in studying BMP signaling that we have not explored is contact-dependent signaling [72, 73], since unlike S2 cells, D17 cells form aggregates with cell-cell contacts [56]. Moreover, the extracellular milieu is a critical factor in the range and efficiency of Dpp signaling [74, 75]. D17 cells exhibit differential expression of several extracellular modulators of BMP signaling, relative to S2 cells [34] (Additional file 3: Table S2). D17 cells express higher levels of dally and dlp which encode heparan sulfate proteoglycans that increase the stability of Dpp and facilitate its transmission in the extracellular environment [72, 76]. Conversely, collagen IV (encoded by $v \mathrm{~kg}$ and $\mathrm{Cg} 25 \mathrm{C}$ ) sequesters Dpp in the ovary and reduces its effectiveness [77]; transcript levels of both $v \mathrm{~kg}$ and $C g 25 \mathrm{C}$ are much lower in D17 cells compared with S2 cells. Finally, a family of related proteins encoded by $t s g, s o g$ and $c v-d$ interact with BMP ligands to shuttle them across long extracellular distances, yet limit local ligand activity [29, 63, 78]; $c v-d$ and $s o g$ have lower expression in D17 cells whereas tsg has low expression in both D17 and S2 cells. Determining which, if any, of these factors contribute to the robust Dpp response we have observed in D17 cells represents an avenue for future research.

\section{Conclusions}

BMP signaling in the publicly available and molecularly characterized D17 cell line is robust. Dpp signal transduction, via contributions from all four BMP receptors, results in the nuclear accumulation of pMad and the transcriptional regulation of known direct target genes such as Dad and brk. Furthermore, we observed transcriptional regulation of genes encoding elements from multiple levels of the BMP signal transduction cascade. In conclusion, we believe that D17 cells offer a naturally responsive, simple, and well-defined in vitro system in which to comprehensively study unresolved aspects of BMP signaling. 


\section{Methods}

\section{Cell culture}

Drosophila ML-DmD4-c1, ML-DmD8, ML-DmD17-c3 (D17), ML-DmBG2-c2, S1 and S2-DRSC (S2) cells $[27,35,37]$ were purchased from the Drosophila Genomics Resource Center (DGRC stocks 126, 92, 107, 53, 9 and 181, respectively). Cells were grown at $25^{\circ}-28^{\circ} \mathrm{C}$ in $\mathrm{M} 3$ Medium (Sigma) supplemented with $10 \%$ heat-inactivated fetal bovine serum (SH30070.02, HyClone), 50 units $/ \mathrm{mL}$ penicillin $\mathrm{G}+50 \mu \mathrm{g} / \mathrm{mL}$ streptomycin sulfate (Gibco) and with (ML-DmD4-c1, ML-DmD8, D17, and ML-DmBG2-c2) or without (S1 and S2) $10 \mu \mathrm{g} / \mathrm{mL}$ human insulin (Sigma). All experiments were performed between cell passages 3-30.

\section{DNA constructs and dsRNA production}

The "dad13" element [38] was amplified by PCR (Primers: Additional file 4: Table S3) and cloned into the luciferase expression vector pGL3 (Promega). This construct is similar in concept, but not identical, to that reported elsewhere [79]. The constitutive CMV-Renilla luciferase construct was used as a transfection control (Promega). For dsRNA production, gene specific DNA sequences were amplified from cDNA by PCR using T7 promoter-containing primers (Additional file 4: Table S3) and cloned into pGEM-T Easy (Promega) for re-amplification. dsRNAs were generated from $1 \mu \mathrm{g}$ PCR template using the MEGAscript T7 kit (Ambion), following the manufacturer's instructions. RNA was pelleted by centrifugation at $>18,000 \times \mathrm{g}$ at $4{ }^{\circ} \mathrm{C}$ for $15 \mathrm{~min}$ and re-suspended in $100-200 \mu \mathrm{L}$ of nuclease free water (Invitrogen). Nucleotides $1-435$ of the GFP coding sequence were used for production of negative control dsRNA.

\section{RNAi and Dpp treatments}

Cells were plated at a density of $2 \times 10^{6}$ cells $/ \mathrm{mL}$ in 24-well plates $(0.5 \mathrm{~mL} /$ well $)$ and allowed to adhere to the bottom of the well $(2 \mathrm{~h})$. Growth medium was removed and serum-free M3 medium containing dsRNA (500 pg - $5 \mu \mathrm{g}$, as indicated) was exchanged daily, for 3 days. Knockdown of target genes was verified by rt-qPCR (Additional file 2: Figure S1a).

Two hours after the final dsRNA treatment the medium was replaced with complete M3 medium, with or without recombinant Dpp (159-DP-020, R\&D Systems) and RNA extractions were performed 18-24 h later. Note that commercial Dpp is provided as a disulfide-bridged homodimer; however, throughout this manuscript we report the effective monomeric concentration.

\section{Luciferase assays}

S2 and D17 cells were plated to a density of $2 \times 10^{6}$ cells $/ \mathrm{mL}$ in 12 -well plates and incubated for $24 \mathrm{~h}$. Transfections were performed using jetPRIME (Polyplus
Transfection). After $24 \mathrm{~h}$, fresh complete media, with or without recombinant Dpp, was added for an additional $24 \mathrm{~h}$. Cells were lysed at room temperature and Firefly/ Renilla luciferase activities were immediately assessed using the Dual Luciferase Reporter Assay System (Promega) and a TD-20/20 Luminometer (Turner Designs).

\section{RNA isolation and rt-qPCR}

RNA was isolated using RNAzol RT (Molecular Research Center, Inc.) and $1 \mu \mathrm{g}$ total RNA was reverse-transcribed using oligo $(\mathrm{dT})_{20}$ and SuperScript III (Invitrogen) under standard conditions. Quantitative PCR on 10 ng cDNA was performed using LightCycler 480 SYBR Green I Master Mix (Roche) according to the manufacturer, and a Bio-Rad CFX 384 Real Time PCR System. Cycling and melt-curve parameters were as follows: $95^{\circ} \mathrm{C} 5 \mathrm{~min}$; $45 \mathrm{cy}$ cles of $95^{\circ} \mathrm{C} 15 \mathrm{~s}, 60^{\circ} \mathrm{C} 15 \mathrm{~s}, 72^{\circ} \mathrm{C}, 15 \mathrm{~s} ; 70$ cycles of $60^{\circ} \mathrm{C}$ $5 \mathrm{~s}+0.5^{\circ} \mathrm{C} /$ cycle. Signals were recorded during the $72^{\circ} \mathrm{C}$ extension phase and at each temperature during the melt analysis. Gene-specific amplification primers are listed in Additional file 4: Table S3. Melting curve analysis was used to confirm that each primer pair produced a single amplicon. The expression of Actin 5C (Act5C) was used as a "housekeeping gene" to normalize expression of genes of interest among samples and treatments. Normalized threshold cycle values were exported to Microsoft Excel and a custom analysis template was used to linearly scale baseline actin expression of control samples among qPCR runs in cases where data were pooled to produce a given figure.

\section{Immunocytochemistry, microscopy and image analysis}

Treated and untreated cells were plated on poly-L-lysine $(0.01 \%)$ coated coverslips in 6-well plates and allowed to settle $(2 \mathrm{~h})$. Cells were fixed (4\% paraformaldehyde in phosphate-buffered saline (PBS) solution), washed (3X) in PBS and permeabilized and blocked in PBS containing $0.1 \%$ Triton X-100 (ThermoFisher) and 5\% normal goat serum (G6767, Sigma) for $30 \mathrm{~min}$ before $1 \mathrm{~h}$ incubation in primary antibody solution. Primary antibodies used in this study were Rabbit-anti-phospho-Smad1/5 (1:250, Cell Signaling) [45] and Mouse-anti-DLG (1:200, Developmental Studies Hybridoma Bank). Cells were washed (2X) in PBS and re-blocked for $15 \mathrm{~min}$, prior to the addition of fluorescently-conjugated secondary antibodies diluted in fresh blocking solution, supplemented with Alexa-546-conjugated phalloidin (1:50; ThermoFisher), for $1 \mathrm{~h}$. Secondary antibodies used were Cy5-goat-anti-mouse IgG and Cy2-goat-anti-rabbit IgG (1:250, Jackson ImmunoResearch). Cells were washed (2X) 5 min in PBS, (1X) $10 \mathrm{~min}$ in PBS containing $0.1 \%$ Triton X-100, and (3X) $5 \mathrm{~min}$ in PBS. Cells were mounted in 2.5\% $(w / v) n$-propyl-gallate dissolved in PBS containing $65 \%(\mathrm{v} / \mathrm{v})$ glycerol, supplemented with 1:10 
Hoechst 33342 (final concentration $10 \mathrm{pg} / \mathrm{mL}$; Sigma) and the coverglass was adhered to a standard microscope slide and sealed with nail polish.

Images were collected with a Leica SPE II confocal system attached to a DM5500Q base using a 40X oil-immersion objective (NA 1.15) with 1.5 zoom factor; insets were collected using a 100X oil-immersion objective (NA 0.70). Laser powers were adjusted to maximize, but not saturate signals in the Dpp-treated samples and were kept constant across all fields (5/coverglass) and slides within an experiment. Representative images are shown and all image adjustments (Leica LASX, Adobe Photoshop) were applied uniformly to all images at a given magnification.

\section{Statistical analysis}

Unless otherwise stated, cell culture experiments consisted of 2 or 3 technical replicates and each experiment was repeated 2 or 3 times. rt-qPCR reactions were performed in triplicate. The average Act5C-normalized expression values were collected for each biological sample and differences between group means were compared using a general linear model in SPSS (v25, IBM). Independent trials were treated as random factors. A posthoc Bonferroni comparison was used to determine pairwise $P$ values. Simple pairwise comparisons of luciferase and rt-qPCR data to determine Dpp treatment effects were analyzed using Student's $t$-test (Microsoft Excel) to compare the means of the treated and untreated samples.

\section{Additional files}

Additional file 1: Table S1. modENCODE gene expression values for core BMP signaling molecules. This table contains the calculated expression values for the indicated genes as originally reported by Cherbas and colleagues (2011). (DOCX 14 kb)

Additional file 2: Figure S1. Validation and titration of RNAi reagents in ML-DmD17-c3 cells. This supplemental figure provides evidence of the efficacy of RNAi treatments in ML-DmD17-c3 cells. (Al $1170 \mathrm{~kb}$ )

Additional file 3: Table S2. modENCODE gene expression values for extracellular modulators of BMP signaling. This table contains the calculated expression values for the indicated genes as originally reported by Cherbas and colleagues (2011). (DOCX $13 \mathrm{~kb}$ )

Additional file 4: Table S3. List of oligonucleotide primers used in this study. Oligonucleotide sequences ( $5^{\prime}$ to $\left.3^{\prime}\right)$ used for the generation of dsRNA and for the assessment of transcript abundance by rt-qPCR. (DOCX $16 \mathrm{~kb}$ )

\section{Abbreviations}

Act5C: Actin at 5C; Bam: Bag of Marbles; BMP: Bone Morphogenetic Protein; Brk: Brinker; Cg25C: Collagen at 25C; Cv-d: Crossveinless-d; D17 cells: MLDmD17-c3 cells; Dad: Daughters against Dpp; DGRC: Drosophila Genomics Resource Center; Dlp: Dally-like protein; Dpp: Decapentaplegic; DRSC: Drosophila RNAi Screening Center; dsRNA: Double-stranded RNA; Gbb: Glass Bottom Boat; KD: Knock-down; Mad: Mothers against Dpp; Med: Medea; p-Mad: Phospho-Mad; Put: Punt; RNAi: RNA interference; rtqPCR: Reverse transcription-quantitative polymerase chain reaction; S2 cells: Schneider line 2 cells; Sax: Saxophone; Scw: Screw; Sog: Short Gastrulation; Tkv: Thickveins; Tsg: Twisted Gastrulation; Vkg: Viking; Wit: Wishful thinking

\section{Acknowledgements}

We thank Karen Gentile of the SUNY-Molecular Analysis Core for training and equipment access for qPCR assays. We also wish to thank Dr. Andrea Viczian and Dana DeSantis for critical comments on this manuscript, and Merin Roy for preliminary rt-qPCR work.

\section{Funding}

This work was funded by NIH grant R01-GM110498 to FP, and supported by an unrestricted grant from Research to Prevent Blindness and funding from Lions District 20-Y. The funding bodies had no part in the design of this study, in the collection, analysis, or interpretation of the data, nor in the writing of this manuscript. Cell stocks were obtained from the Drosophila Genomics Resource Center, which is supported by NIH grant 2P40OD010949.

\section{Availability of data and materials}

All cell lines and antibodies used in this manuscript are available from the sources indicated. Oligonucleotide sequences are included in Additional file 4: Table S3. Raw rt-qPCR data (Bio-Rad CFX output files, or Cq values) are available upon request from the corresponding authors.

\section{Authors' contributions}

Study Conception - DD, FP; Experimental Design - SJN, DD, NJ, FP; Data Analysis - SJN, DD, NJ; Data Interpretation - SJN, DD, NJ, FP; Preparation of Figures - SJN, NJ; Preparation and Revision of Manuscript - SJN, FP; Procurement of Funding - FP. All authors have read and approve of the submitted manuscript.

Ethics approval and consent to participate

Not applicable.

\section{Consent for publication}

Not applicable.

\section{Competing interests}

The authors declare no competing interests.

\section{Publisher's Note}

Springer Nature remains neutral with regard to jurisdictional claims in published maps and institutional affiliations.

\section{Author details}

${ }^{1}$ Center for Vision Research and Department of Ophthalmology, Upstate Medical University, NRB-4610, 505 Irving Ave, Syracuse 13210, NY, USA. ${ }^{2}$ Department of Biochemistry and Molecular Biology, Upstate Medical University, Syracuse, NY, USA. ${ }^{3}$ Department of Neuroscience and Physiology, Upstate Medical University, Syracuse, NY, USA. ${ }^{4}$ Current Address: Department of Surgical Pathology, Yale-New Haven Hospital, New Haven, CT, USA.

Received: 26 November 2018 Accepted: 7 January 2019 Published online: 22 January 2019

\section{References}

1. Heldin $\mathrm{CH}$, Miyazono K, ten Dijke P. TGF-beta signalling from cell membrane to nucleus through SMAD proteins. Nature. 1997;390:465-71.

2. Lochab AK, Extavour CG. Bone morphogenetic protein (BMP) signaling in animal reproductive system development and function. Dev Biol. 2017:427:258-69.

3. Zinski J, Tajer B, Mullins MC. TGF-beta family signaling in early vertebrate development. Cold Spring Harb Perspect Biol. 2018;10.

4. Raftery LA, Korochkina S, Cao J. Smads in Drosophila-interpretation of graded signals in vivo. In Smad Signal Transduction Springer. 2006:55-73.

5. Hamaratoglu F, Affolter M, Pyrowolakis G. Dpp/BMP signaling in flies: from molecules to biology. Semin Cell Dev Biol. 2014;32:128-36.

6. Gelbart WM. The decapentaplegic gene: a TGF-beta homologue controlling pattern formation in Drosophila. Development. 1989;107(Suppl):65-74.

7. Affolter M, Basler K. The decapentaplegic morphogen gradient: from pattern formation to growth regulation. Nat Rev Genet. 2007:8:663-74.

8. Dolezal D, Liu Z, Zhou Q, Pignoni F. Fly LMBR1/LIMR-type protein Lilipod promotes germ-line stem cell self-renewal by enhancing BMP signaling. Proc Natl Acad Sci U S A. 2015;112:13928-33. 
9. Panganiban GE, Rashka KE, Neitzel MD, Hoffmann FM. Biochemical characterization of the Drosophila dpp protein, a member of the transforming growth factor beta family of growth factors. Mol Cell Biol. 1990;10:2669-77.

10. Padgett RW, Johnston RDS, Gelbart WM. A transcript from a Drosophila pattern gene predicts a protein homologous to the transforming growth factor- $\beta$ family. Nature. 1987;325:81-4.

11. Doctor JS, Jackson PD, Rashka KE, Visalli M, Hoffmann FM. Sequence, biochemical characterization, and developmental expression of a new member of the TGF-beta superfamily in Drosophila melanogaster. Dev Biol. 1992;151:491-505.

12. Wharton KA, Thomsen GH, Gelbart WM. Drosophila 60A gene, another transforming growth factor beta family member, is closely related to human bone morphogenetic proteins. Proc Natl Acad Sci U S A. 1991;88:9214-8.

13. Arora K, Levine MS, O'Connor MB. The screw gene encodes a ubiquitously expressed member of the TGF-beta family required for specification of dorsal cell fates in the Drosophila embryo. Genes Dev. 1994;8:2588-601.

14. Massague J, Attisano L, Wrana JL. The TGF-beta family and its composite receptors. Trends Cell Biol. 1994;4:172-8.

15. Wrana JL, Attisano L, Wieser R, Ventura F, Massague J. Mechanism of activation of the TGF-beta receptor. Nature. 1994;370:341-7.

16. Maduzia LL, Padgett RW. Drosophila MAD, a member of the Smad family, translocates to the nucleus upon stimulation of the dpp pathway. Biochem Biophys Res Commun. 1997;238:595-8.

17. Inoue H, Imamura T, Ishidou Y, Takase M, Udagawa Y, Oka Y, Tsuneizumi K, Tabata T, Miyazono K, Kawabata M. Interplay of signal mediators of decapentaplegic (Dpp): molecular characterization of mothers against dpp, Medea, and daughters against dpp. Mol Biol Cell. 1998;9:2145-56.

18. Das P, Maduzia LL, Wang H, Finelli AL, Cho SH, Smith MM, Padgett RW. The Drosophila gene Medea demonstrates the requirement for different classes of Smads in dpp signaling. Development. 1998;125:1519-28.

19. Hudson JB, Podos SD, Keith K, Simpson SL, Ferguson EL. The Drosophila Medea gene is required downstream of $\mathrm{dpp}$ and encodes a functional homolog of human Smad4. Development. 1998;125:1407-20.

20. Wisotzkey RG, Mehra A, Sutherland DJ, Dobens LL, Liu X, Dohrmann C, Attisano L, Raftery LA. Medea is a Drosophila Smad4 homolog that is differentially required to potentiate DPP responses. Development. 1998;125:1433-45.

21. Tsuneizumi K, Nakayama T, Kamoshida Y, Kornberg TB, Christian JL, Tabata T. Daughters against dpp modulates dpp organizing activity in Drosophila wing development. Nature. 1997;389:627-31.

22. Chen D, McKearin D. Dpp signaling silences bam transcription directly to establish asymmetric divisions of germline stem cells. Curr Biol. 2003;13:1786-91.

23. Campbell G, Tomlinson A. Transducing the Dpp morphogen gradient in the wing of Drosophila: regulation of Dpp targets by brinker. Cell. 1999;96:553-62.

24. Jazwinska A, Kirov N, Wieschaus E, Roth S, Rushlow C. The Drosophila gene brinker reveals a novel mechanism of Dpp target gene regulation. Cell. 1999:96:563-73.

25. Minami M, Kinoshita N, Kamoshida $Y$, Tanimoto $H$, Tabata T. brinker is a target of Dpp in Drosophila that negatively regulates Dpp-dependent genes. Nature. 1999:398:242-6.

26. Echalier G. Drosophila cells in culture. New York: Academic Press; 1997.

27. Schneider I. Cell lines derived from late embryonic stages of Drosophila melanogaster. J Embryol Exp Morphol. 1972;27:353-65.

28. Chen HB, Shen J, Ip YT, Xu L. Identification of phosphatases for Smad in the BMP/DPP pathway. Genes Dev. 2006;20:648-53.

29. Chen J, Honeyager SM, Schleede J, Avanesov A, Laughon A, Blair SS. Crossveinless $d$ is a vitellogenin-like lipoprotein that binds BMPs and HSPGs, and is required for normal BMP signaling in the Drosophila wing. Development. 2012;139:2170-6.

30. Kirkpatrick H, Johnson K, Laughon A. Repression of dpp targets by binding of brinker to mad sites. J Biol Chem. 2001;276:18216-22.

31. Shimmi O, O'Connor MB. Physical properties of Tld, Sog, Tsg and Dpp protein interactions are predicted to help create a sharp boundary in bmp signals during dorsoventral patterning of the Drosophila embryo. Development. 2003;130:4673-82.

32. Zeng Z, de Gorter DJ, Kowalski M, ten Dijke P, Shimmi O. Ter94NCP is a novel component involved in BMP signaling. PLoS One. 2014;9:e114475.

33. Van Bortle K, Peterson AJ, Takenaka N, O'Connor MB, Corces VG. CTCFdependent co-localization of canonical Smad signaling factors at architectural protein binding sites in D. Melanogaster. Cell Cycle. 2015;14:2677-87.
34. Cherbas L, Willingham A, Zhang D, Yang L, Zou Y, Eads BD, Carlson JW, Landolin JM, Kapranov P, Dumais J, et al. The transcriptional diversity of 25 Drosophila cell lines. Genome Res. 2011;21:301-14.

35. Ui K, Ueda R, Miyake T. Cell lines from imaginal discs of Drosophila melanogaster. In Vitro Cell Dev Biol. 1987;23:707-11.

36. Celniker SE, Dillon LA, Gerstein MB, Gunsalus KC, Henikoff S, Karpen GH, Kellis M, Lai EC, Lieb JD, MacAlpine DM, et al. Unlocking the secrets of the genome. Nature. 2009;459:927-30.

37. Ui-Tei K, Nishihara S, Sakuma M, Matsuda K, Miyake T, Miyata Y. Chemical analysis of neurotransmitter candidates in clonal cell lines from Drosophila central nervous system. I ACh and L-dopa Neurosci Lett. 1994;174:85-8.

38. Weiss A, Charbonnier E, Ellertsdottir E, Tsirigos A, Wolf C, Schuh R, Pyrowolakis G, Affolter M. A conserved activation element in BMP signaling during Drosophila development. Nat Struct Mol Biol. 2010;17:69-76.

39. Pyrowolakis G, Hartmann B, Muller B, Basler K, Affolter M. A simple molecular complex mediates widespread BMP-induced repression during Drosophila development. Dev Cell. 2004;7:229-40.

40. Chen D, McKearin DM. A discrete transcriptional silencer in the bam gene determines asymmetric division of the Drosophila germline stem cell. Development. 2003;130:1159-70.

41. Gao S, Steffen J, Laughon A. Dpp-responsive silencers are bound by a trimeric mad-Medea complex. J Biol Chem. 2005;280:36158-64.

42. Huang JD, Schwyter DH, Shirokawa JM, Courey AJ. The interplay between multiple enhancer and silencer elements defines the pattern of decapentaplegic expression. Genes Dev. 1993;7:694-704.

43. Ammeux N, Housden BE, Georgiadis A, Hu Y, Perrimon N. Mapping signaling pathway cross-talk in Drosophila cells. Proc Natl Acad Sci U S A. 2016;113:9940-5.

44. Ross JJ, Shimmi O, Vilmos P, Petryk A, Kim H, Gaudenz K, Hermanson S, Ekker SC, O'Connor MB, Marsh JL. Twisted gastrulation is a conserved extracellular BMP antagonist. Nature. 2001;410:479-83.

45. Cao J, Pellock BJ, White K, Raftery LA. A commercial phospho-Smad antibody detects endogenous BMP signaling in Drosophila tissues. Drosophila Information Services. 2006;89:131-5.

46. Clemens JC, Worby CA, Simonson-Leff N, Muda M, Maehama T, Hemmings BA, Dixon JE. Use of double-stranded RNA interference in Drosophila cell lines to dissect signal transduction pathways. Proc Natl Acad Sci U S A. 2000;97:6499-503.

47. Ulvila J, Parikka M, Kleino A, Sormunen R, Ezekowitz RA, Kocks C, Ramet M Double-stranded RNA is internalized by scavenger receptor-mediated endocytosis in Drosophila S2 cells. J Biol Chem. 2006;281:14370-5.

48. Raftery LA, Umulis DM. Regulation of BMP activity and range in Drosophila wing development. Curr Opin Cell Biol. 2012;24:158-65.

49. Kamiya Y, Miyazono K, Miyazawa K. Specificity of the inhibitory effects of dad on TGF-beta family type I receptors, Thickveins, saxophone, and baboon in Drosophila. FEBS Lett. 2008;582:2496-500.

50. Takaesu NT, Bulanin DS, Johnson AN, Orenic TV, Newfeld SJ. A combinatorial enhancer recognized by mad, TCF and Brinker first activates then represses dpp expression in the posterior spiracles of Drosophila. Dev Biol. 2008;313:829-43.

51. Kim D, Kwon YK, Cho KH. The biphasic behavior of incoherent feed-forward loops in biomolecular regulatory networks. Bioessays. 2008;30:1204-11.

52. Kwon YK, Cho KH. Coherent coupling of feedback loops: a design principle of cell signaling networks. Bioinformatics. 2008;24:1926-32.

53. Rogers SL, Rogers GC. Culture of Drosophila S2 cells and their use for RNAimediated loss-of-function studies and immunofluorescence microscopy. Nat Protoc. 2008;3:606-11

54. Cavodeassi F, Rodriguez I, Modolell J. Dpp signalling is a key effector of the wing-body wall subdivision of the Drosophila mesothorax. Development. 2002;129:3815-23.

55. Wang D, Li L, Lu J, Liu S, Shen J. Complementary expression of optomotorblind and the Iroquois complex promotes fold formation to separate wing notum and hinge territories. Dev Biol. 2016;416:225-34.

56. Currie JD, Rogers SL. Using the Drosophila melanogaster D17-c3 cell culture system to study cell motility. Nat Protoc. 2011;6:1632-41.

57. Ayyaz $\mathrm{A}$, Li H, Jasper $\mathrm{H}$. Haemocytes control stem cell activity in the Drosophila intestine. Nat Cell Biol. 2015;17:736-48.

58. Pennetier D, Oyallon J, Morin-Poulard I, Dejean S, Vincent A, Crozatier M. Size control of the Drosophila hematopoietic niche by bone morphogenetic protein signaling reveals parallels with mammals. Proc Natl Acad Sci U S A. 2012;109:3389-94. 
59. Brummel TJ, Twombly V, Marques G, Wrana JL, Newfeld SJ, Attisano L, Massague J, O'Connor MB, Gelbart WM. Characterization and relationship of Dpp receptors encoded by the saxophone and thick veins genes in Drosophila. Cell. 1994;78:251-61.

60. Burke R, Basler K. Dpp receptors are autonomously required for cell proliferation in the entire developing Drosophila wing. Development. 1996;122:2261-9.

61. Haerry TE, Khalsa O, O'Connor MB, Wharton KA. Synergistic signaling by two BMP ligands through the SAX and TKV receptors controls wing growth and patterning in Drosophila. Development. 1998;125:3977-87.

62. Marques G, Bao H, Haerry TE, Shimell MJ, Duchek P, Zhang B, O'Connor MB. The Drosophila BMP type II receptor wishful thinking regulates neuromuscular synapse morphology and function. Neuron. 2002;33:529-43.

63. Shimmi O, Umulis D, Othmer $\mathrm{H}, \mathrm{O}^{\prime} \mathrm{C}$ (onnor MB. Facilitated transport of a $\mathrm{Dpp} / \mathrm{Scw}$ heterodimer by Sog/Tsg leads to robust patterning of the Drosophila blastoderm embryo. Cell. 2005;120:873-86.

64. Nellen D, Affolter M, Basler K. Receptor serine/threonine kinases implicated in the control of Drosophila body pattern by decapentaplegic. Cell. 1994;78:225-37.

65. Neul JL, Ferguson EL. Spatially restricted activation of the SAX receptor by SCW modulates DPP/TKV signaling in Drosophila dorsal-ventral patterning. Cell. 1998:95:483-94.

66. Khalsa O, Yoon JW, Torres-Schumann S, Wharton KA. TGF-beta/BMP superfamily members, Gbb-60A and Dpp, cooperate to provide pattern information and establish cell identity in the Drosophila wing. Development. 1998;125:2723-34.

67. McCabe BD, Marques G, Haghighi AP, Fetter RD, Crotty ML, Haerry TE, Goodman CS, O'Connor MB. The BMP homolog Gbb provides a retrograde signal that regulates synaptic growth at the Drosophila neuromuscular junction. Neuron. 2003;39:241-54.

68. Anderson EN, Wharton KA. Alternative cleavage of the bone morphogenetic protein (BMP), Gbb, produces ligands with distinct developmental functions and receptor preferences. J Biol Chem. 2017;292:19160-78.

69. Ogiso Y, Tsuneizumi K, Masuda N, Sato M, Tabata T. Robustness of the Dpp morphogen activity gradient depends on negative feedback regulation by the inhibitory Smad, dad. Develop Growth Differ. 2011;53:668-78.

70. Pignoni F, Zipursky SL. Induction of Drosophila eye development by decapentaplegic. Development. 1997;124:271-8.

71. Marmion RA, Yakoby N. In locus analysis of patterning evolution of the BMP type II receptor wishful thinking. Development. 2018;145.

72. Dejima K, Kanai MI, Akiyama T, Levings DC, Nakato H. Novel contactdependent bone morphogenetic protein (BMP) signaling mediated by heparan sulfate proteoglycans. J Biol Chem. 2011;286:17103-11.

73. Roy S, Huang H, Liu S, Kornberg TB. Cytoneme-mediated contactdependent transport of the Drosophila decapentaplegic signaling protein. Science. 2014;343:1244624.

74. Sedlmeier G, Sleeman JP. Extracellular regulation of BMP signaling: welcome to the matrix. Biochem Soc Trans. 2017:45:173-81.

75. Wilcockson SG, Sutcliffe C, Ashe HL. Control of signaling molecule range during developmental patterning. Cell Mol Life Sci. 2017;74:1937-56.

76. Fujise M, Takeo S, Kamimura K, Matsuo T, Aigaki T, Izumi S, Nakato H. Dally regulates Dpp morphogen gradient formation in the Drosophila wing. Development. 2003;130:1515-22.

77. Wang X, Harris RE, Bayston LJ, Ashe HL. Type IV collagens regulate BMP signalling in Drosophila. Nature. 2008;455:72-7.

78. Shimmi O, Ralston A, Blair SS, O'Connor MB. The crossveinless gene encodes a new member of the twisted gastrulation family of BMP-binding proteins which, with short gastrulation, promotes BMP signaling in the crossveins of the Drosophila wing. Dev Biol. 2005;282:70-83.

79. Li W, Li W, Zou L, Ji S, Li C, Liu K, Zhang G, Sun Q, Xiao F, Chen D. Membrane targeting of inhibitory Smads through palmitoylation controls TGF-beta/BMP signaling. Proc Natl Acad Sci U S A. 2017;114:13206-11.

Ready to submit your research? Choose BMC and benefit from:

- fast, convenient online submission

- thorough peer review by experienced researchers in your field

- rapid publication on acceptance

- support for research data, including large and complex data types

- gold Open Access which fosters wider collaboration and increased citations

- maximum visibility for your research: over $100 \mathrm{M}$ website views per year

At BMC, research is always in progress.

Learn more biomedcentral.com/submissions 